\title{
STRONGLY GORENSTEIN FLAT MODULES
}

\author{
NANQING DING ${ }^{凶}$, YUANLIN LI and LIXIN MAO
}

\author{
(Received 28 September 2007; accepted 2 March 2008)
}

Communicated by J. Du

Dedicated to Professor Boxun Zhou on the occasion of his 90th birthday and Professor Wenting Tong on his 70th birthday

\begin{abstract}
In this paper, strongly Gorenstein flat modules are introduced and investigated. An $R$-module $M$ is called strongly Gorenstein flat if there is an exact sequence $\cdots \rightarrow P_{1} \rightarrow P_{0} \rightarrow P^{0} \rightarrow P^{1} \rightarrow \cdots$ of projective $R$-modules with $M=\operatorname{ker}\left(P^{0} \rightarrow P^{1}\right)$ such that $\operatorname{Hom}(-, F)$ leaves the sequence exact whenever $F$ is a flat $R$-module. Several well-known classes of rings are characterized in terms of strongly Gorenstein flat modules. Some examples are given to show that strongly Gorenstein flat modules over coherent rings lie strictly between projective modules and Gorenstein flat modules. The strongly Gorenstein flat dimension and the existence of strongly Gorenstein flat precovers and pre-envelopes are also studied.
\end{abstract}

2000 Mathematics subject classification: primary 16D40; secondary 16E05, 18 G20.

Keywords and phrases: strongly Gorenstein flat module, Gorenstein flat module, precover, pre-envelope, cotorsion theory.

\section{Introduction}

Let $R$ be a ring. A right $R$-module $N$ is called Gorenstein flat [15] if there is an exact sequence $\cdots \rightarrow F_{1} \rightarrow F_{0} \rightarrow F^{0} \rightarrow F^{1} \rightarrow \cdots$ of flat right $R$-modules with $N=\operatorname{ker}\left(F^{0} \rightarrow F^{1}\right)$ such that $-\otimes E$ leaves the sequence exact whenever $E$ is an injective left $R$-module. Gorenstein flat modules have been studied extensively by many authors (see, for example, $[5,7,8,10,12-16,19,20,23])$. These modules have nice properties when the ring in question is $n$-Gorenstein (a ring $R$ is called $n$ Gorenstein if $R$ is a left and right Noetherian ring with self-injective dimension at most $n$ for an integer $n \geq 0$ on either side).

Following [22], a left $R$-module $M$ is called $F P$-injective if $\operatorname{Ext}^{1}(N, M)=0$ for all finitely presented left $R$-modules $N$. The $F P$-injective dimension of $M$,

This research was partially supported by SRFDP (No. 20050284015), NSFC (No. 10771096), a discovery grant from NSERC, Science Research Fund of Nanjing Institute of Technology (KXJ07061), Jiangsu 333 Project, and Jiangsu Qinglan Project.

(C) 2009 Australian Mathematical Society 1446-7887/2009 $\$ 16.00$ 
denoted by $F P$-id $(M)$, is defined to be the smallest nonnegative integer $n$ such that $\operatorname{Ext}^{n+1}(F, M)=0$ for every finitely presented left $R$-module $F$. If no such $n$ exists, set $F P$-id $(M)=\infty$. A ring $R$ is said to be an $n-F C$ ring [10] if $R$ is a left and right coherent ring with $F P-\mathrm{id}\left({ }_{R} R\right) \leq n$ and $F P-\mathrm{id}\left(R_{R}\right) \leq n$ for an integer $n \geq 0$. A ring $R$ is called an $F C$ ring if $R$ is $0-F C$.

It is clear that every $n$-Gorenstein ring is $n-F C$, but the converse is not true in general. For example, let $R=\Pi F$, an infinite product of a field $F$; then $R$ is a commutative von Neumann regular ring. Clearly, $R$ is $n-F C$ but not $n$-Gorenstein for any $n \geq 0$. Furthermore, if $S=R\left[X_{1}, X_{2}, \ldots, X_{m}\right]$, the ring of polynomials in $m$ indeterminates over $R$, then $S$ is $n-F C$ but not $n$-Gorenstein for all $n \geq m$ (see [18, Theorem 7.3.1]).

The main purpose of this paper is to introduce and study strongly Gorenstein flat modules over $n-F C$ rings. In Section 2, the definition and some general results are given. A right $R$-module $M$ is called strongly Gorenstein flat if there is an exact sequence $\cdots \rightarrow P_{1} \rightarrow P_{0} \rightarrow P^{0} \rightarrow P^{1} \rightarrow \cdots$ of projective right $R$-modules with $M=\operatorname{ker}\left(P^{0} \rightarrow P^{1}\right)$ such that $\operatorname{Hom}(-, \mathcal{F}$ lat $)$ leaves the sequence exact, where $\mathcal{F}$ lat stands for the class of all flat right $R$-modules. It is proven that a right $R$-module $M$ over a left coherent ring $R$ is strongly Gorenstein flat if and only if $M$ has an exact right $\mathcal{F}$ lat-resolution and $\operatorname{Ext}^{i}(M, F)=0$ for all flat right $R$-modules $F$ and all $i \geq 1$. For an $n-F C$ ring $R$, we prove that $w D(R) \leq n$ if and only if every strongly Gorenstein flat right $R$-module is projective (flat). Several well-known classes of rings are characterized in terms of strongly Gorenstein flat modules. For instance, $R$ is a right perfect ring if and only if every flat right $R$-module is strongly Gorenstein flat; $R$ is a $Q F$ ring if and only if every right $R$-module is strongly Gorenstein flat. Some examples are given to show that strongly Gorenstein flat modules over coherent rings lie strictly between projective modules and Gorenstein flat modules.

In Section 3, we define and investigate the strongly Gorenstein flat dimension for modules and rings. Given a right $R$-module $M$, the strongly Gorenstein flat dimension $S G f d(M)$ of $M$ is defined to be the infimum of the set of $n$ such that there exists an exact sequence $0 \rightarrow G_{n} \rightarrow \cdots \rightarrow G_{1} \rightarrow G_{0} \rightarrow M \rightarrow 0$ of right $R$-modules, where each $G_{i}$ is strongly Gorenstein flat. If no such $n$ exists, set $\operatorname{SGf} d(M)=\infty$. The right strongly Gorenstein flat dimension $\operatorname{rSGFD}(R)$ of a ring $R$ is defined to be the supremum of the set of $S G f d(M)$ such that $M$ is any right $R$-module. It is shown that $r F I D(R) \leq r S G F D(R) \leq r D(R)$ for any ring $R$ and the equalities hold if $w D(R)<\infty$, where $r F I D(R)$ is defined to be the supremum of the set of $\operatorname{id}(M)$ such that $M$ is any flat right $R$-module. For a two-sided coherent ring $R$ and an integer $n \geq 1$, we get that $R$ is an $n-F C$ ring if and only if $S G f d(M) \leq n$ for any (right and

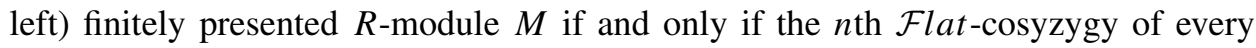
(right and left) finitely presented $R$-module is strongly Gorenstein flat.

Section 4 is devoted to the existence of precovers and pre-envelopes by strongly Gorenstein flat modules. Let $\mathcal{S G \mathcal { F }}$ be the class of all strongly Gorenstein flat right $R$-modules and $\mathcal{I}_{n}$ the class of all right $R$-modules of injective dimension at most $n$, where $n$ is a fixed nonnegative integer. We show that, for a left coherent ring $R$ 


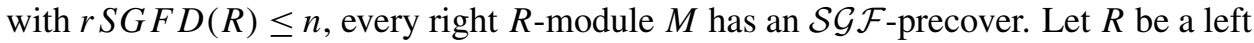
coherent ring with $F P$-id $\left({ }_{R} R\right) \leq n$. It is shown that $r S G F D(R) \leq n$ if and only if $r F I D(R) \leq n$ if and only if $\left(\mathcal{S G \mathcal { F }}, \mathcal{I}_{n}\right)$ is a cotorsion theory. Finally, for an $n-F C$ ring

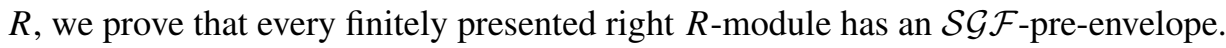

Next we recall some known notions and facts required in the paper. Let $\mathcal{C}$ be a class of $R$-modules and $M$ an $R$-module. Following [11], we say that a homomorphism $\phi: M \rightarrow C$ is a $\mathcal{C}$-pre-envelope if $C \in \mathcal{C}$ and the abelian group homomorphism $\operatorname{Hom}\left(\phi, C^{\prime}\right): \operatorname{Hom}\left(C, C^{\prime}\right) \rightarrow \operatorname{Hom}\left(M, C^{\prime}\right)$ is surjective for each $C^{\prime} \in \mathcal{C}$. Dually we have the definition of a $\mathcal{C}$-precover.

Given a class $\mathcal{L}$ of $R$-modules, write $\mathcal{L}^{\perp}=\left\{C \mid \operatorname{Ext}^{1}(L, C)=0\right.$ for all $\left.L \in \mathcal{L}\right\}$ and ${ }^{\perp} \mathcal{L}=\left\{C \mid \operatorname{Ext}^{1}(C, L)=0\right.$ for all $\left.L \in \mathcal{L}\right\}$. A pair $(\mathcal{F}, \mathcal{C})$ of classes of $R$-modules is called a cotorsion theory [13] if $\mathcal{F}^{\perp}=\mathcal{C}$ and $\mathcal{F}={ }^{\perp} \mathcal{C}$.

It is known that a left coherent ring is characterized by the condition that every right $R$-module has a $\mathcal{F l a t}$-pre-envelope (see [11]). So, for a left coherent ring $R$, every right $R$-module $M$ has a right $\mathcal{F}$ lat-resolution, that is, there is a $\operatorname{Hom}(-, \mathcal{F}$ lat $)$ exact complex $0 \rightarrow M \rightarrow F^{0} \rightarrow F^{1} \rightarrow \cdots$ (not necessarily exact) with each $F^{i}$ flat. Let $L^{0}=M, L^{1}=\operatorname{coker}\left(M \rightarrow F^{0}\right), L^{i}=\operatorname{coker}\left(F^{i-2} \rightarrow F^{i-1}\right)$ for $i \geq 2$. The $n$th cokernel $L^{n}(n \geq 0)$ is called the $n$th $\mathcal{F l a t}$-cosyzygy of $M$. Note that we can choose each $F^{i}$ to be finitely generated projective if $M$ is finitely presented by [13, Example 8.3.10]. So by the $n$th $\mathcal{F l a t}$-cosyzygy of a finitely presented right $R$-module, we will mean the $n$th cokernel in such a right $\mathcal{F l a t}$-resolution.

On the other hand, it has been proven that every right $R$-module over any ring $R$ has a $\mathcal{F l a t}$-precover (see [6]), and hence every right $R$-module $N$ has a left $\mathcal{F}$ lat resolution, that is, there is a $\operatorname{Hom}(\mathcal{F}$ lat, -$)$ exact complex $\cdots \rightarrow F_{1} \rightarrow F_{0} \rightarrow N \rightarrow 0$ with each $F_{i}$ flat. Thus, for a left coherent ring $R$, $\operatorname{Hom}(-,-)$ is left balanced on $\mathcal{M}_{\mathcal{R}} \times \mathcal{M}_{\mathcal{R}}$ by $\mathcal{F l a t} \times \mathcal{F}$ lat (see [13, Definition 8.2.13]), where $\mathcal{M}_{\mathcal{R}}$ denotes the category of right $R$-modules. So the $n$th left derived functor, which is denoted by $\operatorname{Ext}_{n}(-,-)$, can be computed using a right $\mathcal{F l a t}$-resolution of the first variable or a left $\mathcal{F}$ lat-resolution of the second variable.

Throughout this paper, $R$ is an associative ring with identity and all modules are unitary. Then $r D(R)(w D(R))$ stands for the right (the weak) global dimension of $R$. We denote by $M_{R}\left({ }_{R} M\right)$ a right (left) $R$-module. The character module $\operatorname{Hom}_{\mathbb{Z}}(M, \mathbb{Q} / \mathbb{Z})$ is denoted by $M^{+} ; \operatorname{fd}(M), \operatorname{id}(M)$ and $\operatorname{pd}(M)$ stand for the flat, injective and projective dimensions of $M$, respectively. Let $M$ and $N$ be two $R$ modules. Then $\operatorname{Hom}(M, N)\left(\operatorname{Ext}^{n}(M, N)\right)$ means $\operatorname{Hom}_{R}(M, N)\left(\operatorname{Ext}_{R}^{n}(M, N)\right)$, and similarly $M \otimes N\left(\operatorname{Tor}_{n}(M, N)\right)$ denotes $M \otimes_{R} N\left(\operatorname{Tor}_{n}^{R}(M, N)\right)$ for an integer $n \geq 1$. For unexplained definitions and terminology, we refer the reader to $[1,13,21,23]$.

\section{Strongly Gorenstein flat modules}

We start with the following result.

Definition 2.1. A right $R$-module $M$ is called strongly Gorenstein flat if there is an exact sequence $\cdots \rightarrow P_{1} \rightarrow P_{0} \rightarrow P^{0} \rightarrow P^{1} \rightarrow \cdots$ of projective right $R$-modules with $M=\operatorname{ker}\left(P^{0} \rightarrow P^{1}\right)$ such that $\operatorname{Hom}(-, \mathcal{F l a t})$ leaves the sequence exact. 
REMARK 2.2.

(1) It is clear that every projective module is strongly Gorenstein flat.

(2) The class of strongly Gorenstein flat right $R$-modules is closed under direct sums by definition.

(3) If $\mathcal{P}=\cdots \rightarrow P_{1} \rightarrow P_{0} \rightarrow P^{0} \rightarrow P^{1} \rightarrow \cdots$ is a $\operatorname{Hom}(-, \mathcal{F}$ lat $)$ exact exact sequence of projective right $R$-modules, then by symmetry, all the images, the kernels and the cokernels of $\mathcal{P}$ are strongly Gorenstein flat.

The next proposition shows that strongly Gorenstein flat modules over coherent rings are indeed stronger than Gorenstein flat modules.

PROPOSITION 2.3. Let $R$ be a left coherent ring. Then every strongly Gorenstein flat right $R$-module is Gorenstein flat.

Proof. Let $M$ be a strongly Gorenstein flat right $R$-module. Then there is an exact sequence $\cdots \rightarrow P_{1} \rightarrow P_{0} \rightarrow P^{0} \rightarrow P^{1} \rightarrow \cdots$ of projective right $R$-modules with $M=\operatorname{ker}\left(P^{0} \rightarrow P^{1}\right)$ such that $\operatorname{Hom}(-, \mathcal{F l a t})$ leaves the sequence exact. Let $E$ be an injective left $R$-module. Then $E^{+}$is flat by [17, Theorem 2.2]. So we get the exact sequence

$\cdots \rightarrow \operatorname{Hom}\left(P^{1}, E^{+}\right) \rightarrow \operatorname{Hom}\left(P^{0}, E^{+}\right) \rightarrow \operatorname{Hom}\left(P_{0}, E^{+}\right) \rightarrow \operatorname{Hom}\left(P_{1}, E^{+}\right) \rightarrow \cdots$

which gives the exactness of

$$
\cdots \rightarrow\left(P^{1} \otimes E\right)^{+} \rightarrow\left(P^{0} \otimes E\right)^{+} \rightarrow\left(P_{0} \otimes E\right)^{+} \rightarrow\left(P_{1} \otimes E\right)^{+} \rightarrow \cdots .
$$

Thus we have the exact sequence

$$
\cdots \rightarrow P_{1} \otimes E \rightarrow P_{0} \otimes E \rightarrow P^{0} \otimes E \rightarrow P^{1} \otimes E \rightarrow \cdots .
$$

Hence $M$ is Gorenstein flat.

LEMMA 2.4. Let $M$ be a strongly Gorenstein flat right $R$-module. Then:

(1) $\operatorname{Ext}^{i}(M, G)=0$ for all right $R$-modules $G$ with $\operatorname{fd}(G)<\infty$ and all $i \geq 1$;

(2) either $M$ is projective or $\mathrm{fd}(M)=\infty$.

Proof. (1) By hypothesis, there is a $\operatorname{Hom}(-, \mathcal{F}$ lat $)$ exact exact sequence $\cdots \rightarrow$ $P_{1} \rightarrow P_{0} \rightarrow M \rightarrow 0$ with each $P_{i}$ projective. Thus $\operatorname{Ext}^{i}(M, F)=0$ for all flat right $R$-modules $F$ and all $i \geq 1$. By dimension shifting, $\operatorname{Ext}^{i}(M, G)=0$ for all right $R$ modules $G$ with $\operatorname{fd}(G)<\infty$ and all $i \geq 1$.

(2) Suppose $\operatorname{fd}(M)<\infty$. We may assume that $\operatorname{fd}(M) \leq n$ with $1 \leq n<\infty$. Then there is a $\operatorname{Hom}(-, \mathcal{F}$ lat $)$ exact exact sequence $0 \rightarrow F_{n} \rightarrow P_{n-1} \rightarrow \cdots \rightarrow P_{1} \rightarrow$ $P_{0} \rightarrow M \rightarrow 0$ with $F_{n}$ flat and each $P_{i}$ projective. It is easy to see that $M$ is isomorphic to a direct summand of $P_{0}$, and hence $M$ is projective.

Corollary 2.5. A right $R$-module $M$ is projective if and only if $M$ is flat and strongly Gorenstein flat.

Now we give some characterizations of strongly Gorenstein flat modules. 
THEOREM 2.6. Let $R$ be a left coherent ring. Then the following statements are equivalent for a right $R$-module $M$.

(1) $M$ is strongly Gorenstein flat.

(2) $M$ has an exact right $\mathcal{F l a t - r e s o l u t i o n}$ and $\operatorname{Ext}^{i}(M, F)=0$ for all right $R$-modules $F$ with $\mathrm{fd}(F)<\infty$ and all $i \geq 1$.

(3) $M$ has an exact right $\mathcal{F l a t - r e s o l u t i o n ~ a n d ~} \operatorname{Ext}^{i}(M, F)=0$ for all flat right $R$-modules $F$ and all $i \geq 1$.

Moreover, if $F \mathrm{P}-\mathrm{id}\left({ }_{R} R\right)<\infty$, then the above conditions are equivalent to

(4) $\operatorname{Ext}^{i}(M, F)=0$ for all flat right $R$-modules $F$ and all $i \geq 1$.

PROOF. That (1) implies (2) follows from Lemma 2.4(1).

That (2) implies (3) is trivial.

To show that (3) implies (1), let $f: M \rightarrow F^{0}$ be a $\mathcal{F l a t}$-pre-envelope of $M$. Consider the short exact sequence $0 \rightarrow K^{0} \rightarrow P^{0} \stackrel{\pi}{\rightarrow} F^{0} \rightarrow 0$ with $P^{0}$ projective. There exists $g: M \rightarrow P^{0}$ such that $\pi g=f$ since $K^{0}$ is flat and $\operatorname{Ext}^{1}\left(M, K^{0}\right)=0$. It is easy to verify that $g: M \rightarrow P^{0}$ is a $\mathcal{F l a t}$-pre-envelope. Thus, for any flat right $R$-module $F$, there is the exact sequence

$$
\operatorname{Hom}\left(P^{0}, F\right) \rightarrow \operatorname{Hom}(\operatorname{im}(g), F) \rightarrow 0 .
$$

In addition, the exactness of $0 \rightarrow \operatorname{im}(g) \rightarrow P^{0} \rightarrow \operatorname{coker}(g) \rightarrow 0$ yields the exact sequence

$$
\operatorname{Hom}\left(P^{0}, F\right) \rightarrow \operatorname{Hom}(\operatorname{im}(g), F) \rightarrow \operatorname{Ext}^{1}(\operatorname{coker}(g), F) \rightarrow 0 .
$$

Hence $\operatorname{Ext}^{1}(\operatorname{coker}(g), F)=0$. So coker $(g)$ has a $\mathcal{F l a t}$-pre-envelope $\operatorname{coker}(g) \rightarrow P^{1}$ with $P^{1}$ projective by the proof above. Continuing this process, we can get a $\operatorname{Hom}(-, \mathcal{F}$ lat $)$ exact complex $0 \rightarrow M \rightarrow P^{0} \rightarrow P^{1} \rightarrow \cdots$ with each $P^{i}$ projective. Note that $\operatorname{Ext}_{i}\left(M,\left({ }_{R} R\right)^{+}\right)=0$ for all $i \geq 1$ and $\left.\operatorname{Ext}_{0}\left(M,{ }_{R} R\right)^{+}\right) \cong M^{+}$since $M$ has an exact right $\mathcal{F}$ lat-resolution and $\left({ }_{R} R\right)^{+}$is injective. So the complex $0 \rightarrow M \rightarrow$ $P^{0} \rightarrow P^{1} \rightarrow \cdots$ is exact since $\left({ }_{R} R\right)^{+}$is a cogenerator. On the other hand, there is a $\operatorname{Hom}(-, \mathcal{F}$ lat $)$ exact exact sequence $\cdots \rightarrow P_{1} \rightarrow P_{0} \rightarrow M \rightarrow 0$ with each $P_{i}$ projective since $\operatorname{Ext}^{i}(M, F)=0$ for all flat right $R$-modules $F$ and all $i \geq 1$. Now we get a $\operatorname{Hom}(-, \mathcal{F}$ lat $)$ exact exact sequence $\cdots \rightarrow P_{1} \rightarrow P_{0} \rightarrow P^{0} \rightarrow P^{1} \rightarrow \cdots$ of projective right $R$-modules with $M \cong \operatorname{coker}\left(P_{1} \rightarrow P_{0}\right)$. So $M$ is strongly Gorenstein flat.

That (3) implies (4) is trivial.

Finally, we prove that (4) implies (1). By the proof that (3) implies (1), we obtain a $\operatorname{Hom}(-, \mathcal{F l a t})$ exact complex

$$
\mathcal{E}=\cdots \rightarrow P_{1} \rightarrow P_{0} \rightarrow P^{0} \rightarrow P^{1} \rightarrow \cdots
$$

of projective right $R$-modules such that $M \cong \operatorname{coker}\left(P_{1} \rightarrow P_{0}\right)$. Next we will show that $\operatorname{Hom}(\mathcal{E}, N)$ is exact for any right $R$-module $N$ with $\operatorname{fd}(N)=n<\infty$. We proceed 
by induction on $n$. The case $n=0$ is clear. Let $n \geq 1$. There is an exact sequence $0 \rightarrow K \rightarrow P \rightarrow N \rightarrow 0$ with $P$ projective, which induces an exact sequence

$$
0 \rightarrow \operatorname{Hom}(\mathcal{E}, K) \rightarrow \operatorname{Hom}(\mathcal{E}, P) \rightarrow \operatorname{Hom}(\mathcal{E}, N) \rightarrow 0
$$

of complexes. Note that $\operatorname{fd}(K)=n-1$, so $\operatorname{Hom}(\mathcal{E}, K)$ is exact by induction. Thus $\operatorname{Hom}(\mathcal{E}, N)$ is exact by [21, Theorem 6.3]. In particular, $\operatorname{Hom}\left(\mathcal{E},\left({ }_{R} R\right)^{+}\right)$is exact since $\operatorname{fd}\left(\left({ }_{R} R\right)^{+}\right)=F P-\operatorname{id}\left({ }_{R} R\right)<\infty$ by [17, Theorem 2.2]. Therefore $\mathcal{E}$ is an exact sequence. So $M$ is strongly Gorenstein flat.

REMARK 2.7. By the proof that (3) implies (1) in Theorem 2.6, any strongly Gorenstein flat right $R$-module $M$ over a left coherent ring $R$ has a $\mathcal{F l a t}$-pre-envelope $\alpha: M \rightarrow F$ with $F$ projective. Moreover, $\operatorname{coker}(\alpha)$ is still strongly Gorenstein flat.

Recall that a right $R$-module $M$ is called Gorenstein projective [12] if there is an exact sequence $\cdots \rightarrow P_{1} \rightarrow P_{0} \rightarrow P^{0} \rightarrow P^{1} \rightarrow \cdots$ of projective right $R$ modules with $M=\operatorname{ker}\left(P^{0} \rightarrow P^{1}\right)$ and such that $\operatorname{Hom}(-, P)$ leaves the sequence exact whenever $P$ is a projective right $R$-module.

COROLlary 2.8. Let $R$ be an $n$-Gorenstein ring. Then the class of strongly Gorenstein flat right $R$-modules coincides with the class of Gorenstein projective right $R$-modules.

PROOF. By definition, every strongly Gorenstein flat right $R$-module is Gorenstein projective.

Conversely, let $M$ be a Gorenstein projective right $R$-module. Then, by [13, Remark 10.2.2], $\operatorname{Ext}^{i}(M, L)=0$ for all $i \geq 1$ and all right $R$-modules $L$ of finite projective dimension. For any flat right $R$-module $N$, we have $\operatorname{pd}(N)<\infty$ by [13, Proposition 9.1.2]. So $\operatorname{Ext}^{i}(M, N)=0$ for all $i \geq 1$. Thus $M$ is strongly Gorenstein flat by Theorem 2.6.

COROLlary 2.9. Let $n$ be a fixed nonnegative integer. The following statements are equivalent for a left coherent ring $R$.

(1) Every nth $\mathcal{F}$ lat-cosyzygy of any right $R$-module is strongly Gorenstein flat.

(2) $\quad F P-\operatorname{id}\left({ }_{R} R\right) \leq n$ and $\operatorname{Ext}^{i}\left(L^{n}, F\right)=0$ for all $n$th $\mathcal{F}$ lat-cosyzygies $L^{n}$ of right $R$-modules, all flat right $R$-modules $F$ and all $i \geq 1$.

Proof. To prove that (1) implies (2), it is enough to show that $F P-\operatorname{id}\left({ }_{R} R\right) \leq n$. There is a $\operatorname{Hom}(\mathcal{F}$ lat,-$)$ exact exact sequence $0 \rightarrow K_{n} \stackrel{\alpha}{\rightarrow} F_{n-1} \stackrel{\varphi}{\rightarrow} F_{n-2} \rightarrow \cdots \rightarrow$ $F_{1} \rightarrow F_{0} \rightarrow\left({ }_{R} R\right)^{+} \rightarrow 0$ with each $F_{i}$ flat. Let $\beta: F_{n} \rightarrow K_{n}$ be a $\mathcal{F l a t}$-precover of $K_{n}$. Since the $n$th $\mathcal{F l a t}$-cosyzygy of $K_{n}$ is strongly Gorenstein projective, $\operatorname{Ext}_{n-1}\left(K_{n},\left({ }_{R} R\right)^{+}\right)=0$. So

$$
\operatorname{Hom}\left(K_{n}, F_{n}\right) \stackrel{(\alpha \beta)_{*}}{\rightarrow} \operatorname{Hom}\left(K_{n}, F_{n-1}\right) \stackrel{\varphi_{*}}{\rightarrow} \operatorname{Hom}\left(K_{n}, F_{n-2}\right)
$$


is exact. Since $\alpha \in \operatorname{ker}\left(\varphi_{*}\right)=\operatorname{im}(\alpha \beta)_{*}$, there exists $\gamma \in \operatorname{Hom}\left(K_{n}, F_{n}\right)$ such that $\alpha=\alpha \beta \gamma$. Thus $\beta \gamma=1$, and hence $K_{n}$ is flat. So $\operatorname{fd}\left(\left({ }_{R} R\right)^{+}\right) \leq n$. It follows that $F P-\operatorname{id}\left({ }_{R} R\right)=\operatorname{fd}\left(\left({ }_{R} R\right)^{+}\right) \leq n$ by [17, Theorem 2.2].

The reverse implication follows from Theorem 2.6.

Proposition 2.10. Let $R$ be a left coherent ring and $0 \rightarrow A \rightarrow B \rightarrow C \rightarrow 0$ an exact sequence of right $R$-modules.

(1) If $A$ and $C$ are strongly Gorenstein flat, then so is $B$.

(2) If $B$ and $C$ are strongly Gorenstein flat, then so is $A$.

(3) If $A$ and $B$ are strongly Gorenstein flat, then $C$ is strongly Gorenstein flat if and only if $\operatorname{Ext}^{1}(C, F)=0$ for all flat right $R$-modules $F$.

Thus the class of strongly Gorenstein flat right R-modules is closed under direct summands.

Proof. If $C$ is strongly Gorenstein flat, then $\operatorname{Ext}^{1}(C, F)=0$ for all flat right $R$-modules $F$ by Lemma 2.4. But the fact that $\operatorname{Ext}^{1}(C, F)=0$ for all flat right $R$ modules $F$ implies that $0 \rightarrow A \rightarrow B \rightarrow C \rightarrow 0$ is $\operatorname{Hom}(-, \mathcal{F}$ lat $)$ exact. Thus, by [13, Theorem 8.2.5(2)], we obtain the long exact sequence

$$
\begin{aligned}
\cdots & \rightarrow \operatorname{Ext}_{n}\left(A,\left({ }_{R} R\right)^{+}\right) \rightarrow \operatorname{Ext}_{n-1}\left(C,\left(_{R} R\right)^{+}\right) \rightarrow \operatorname{Ext}_{n-1}\left(B,\left({ }_{R} R\right)^{+}\right) \\
& \rightarrow \operatorname{Ext}_{n-1}\left(A,\left({ }_{R} R\right)^{+}\right) \rightarrow \cdots \rightarrow \operatorname{Ext}_{1}\left(A,\left({ }_{R} R\right)^{+}\right) \rightarrow \operatorname{Ext}_{0}\left(C,\left({ }_{R} R\right)^{+}\right) \\
& \rightarrow \operatorname{Ext}_{0}\left(B,\left({ }_{R} R\right)^{+}\right) \rightarrow \operatorname{Ext}_{0}\left(A,\left({ }_{R} R\right)^{+}\right) \rightarrow 0 .
\end{aligned}
$$

So (1), (2) and (3) follow from Theorem 2.6.

The last statement holds by (1), (2), Remark 2.2(2) and [20, Proposition 1.4].

REMARK 2.11. We note that [13, Theorem 11.5.6] is an immediate consequence of Proposition 2.10 by Corollary 2.8 .

Recall that $R$ is a right perfect ring [4] if every right $R$-module has a projective cover. It is well known that $R$ is right perfect if and only if every flat right $R$-module is projective.

COROLlary 2.12. Let $R$ be a left coherent and right perfect ring, $n$ a fixed nonnegative integer and $F P-\operatorname{id}\left({ }_{R} R\right) \leq n$. The following statements are equivalent for a right $R$-module $M$.

(1) $M$ is strongly Gorenstein flat.

(2) $M$ is Gorenstein projective.

(3) $M$ is Gorenstein flat.

Proof. That (1) holds if and only if (2) holds is clear since $R$ is right perfect.

That (1) implies (3) follows from Proposition 2.3.

To prove that (3) implies (1), let $F$ be a flat right $R$-module and $i \geq 1$. Then there is a pure exact sequence $0 \rightarrow F \rightarrow F^{++} \rightarrow L \rightarrow 0$. Note that $F^{+}$is injective, 
and hence $F^{++}$is flat since $R$ is left coherent. Thus $L$ is flat and so projective since $R$ is right perfect. It follows that $F$ is isomorphic to a direct summand of $F^{++}$. Note that $\operatorname{Ext}^{i}\left(M, F^{++}\right) \cong \operatorname{Tor}_{i}\left(M, F^{+}\right)^{+}$and $\operatorname{Tor}_{i}\left(M, F^{+}\right)=0$ by (3). Thus $\operatorname{Ext}^{i}\left(M, F^{++}\right)=0$, and so $\operatorname{Ext}^{i}(M, F)=0$. Hence $M$ is strongly Gorenstein flat by Theorem 2.6.

LEMMA 2.13. Let $R$ be an $n-F C$ ring. Consider the following conditions for a right $R$-module $M$.

(1) $M$ is strongly Gorenstein flat.

(2) $M$ is Gorenstein projective.

(3) $M$ is Gorenstein flat.

Then (1) implies (2) and (2) implies (3). If M is finitely presented, then (3) implies (1).

Proof. That (1) implies (2) is trivial. That (2) implies (3) follows from [10, Theorem 5].

To prove that (3) implies (1), let $F$ be a flat right $R$-module and $i \geq 1$. Since $R$ is right coherent and $M$ is finitely presented, there is a standard isomorphism $\operatorname{Ext}^{i}(M, F)^{+} \cong \operatorname{Tor}_{i}\left(M, F^{+}\right)$by [21, Theorem 9.51] and the remark following it. But $\operatorname{Tor}_{i}\left(M, F^{+}\right)=0$ by (3). Thus $\operatorname{Ext}^{i}(M, F)^{+}=0$ and so $\operatorname{Ext}^{i}(M, F)=0$. Hence $M$ is strongly Gorenstein flat by Theorem 2.6.

PROPOSITION 2.14. The following statements are equivalent for an $n-F C$ ring $R$.

(1) $w D(R) \leq n$.

(2) Every Gorenstein flat right $R$-module is flat.

(3) Every strongly Gorenstein flat right $R$-module is projective.

(4) Every strongly Gorenstein flat right $R$-module is flat.

PROOF. That (1) holds if and only (2) holds follows from [10, Theorem 13].

To prove that (2) implies (3), let $M$ be a strongly Gorenstein flat right $R$-module. Then $M$ is Gorenstein flat by Proposition 2.3. So $M$ is flat by (2), and hence $M$ is projective by Corollary 2.5 .

That (3) implies (4) is trivial.

To prove that (4) implies (2), let $M$ be a Gorenstein flat right $R$-module. Then, by [10, Theorem 5], $M \cong \lim M_{i}$ for some inductive system $\left(\left(M_{i}\right),\left(f_{i j}\right)\right)$, where each

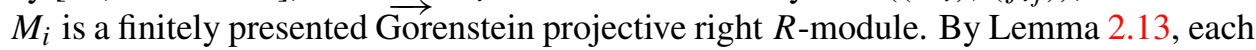
$M_{i}$ is strongly Gorenstein flat, and so flat by (4). Thus $M$ is flat.

PROPOSITION 2.15. The following statements are equivalent for a ring $R$.

(1) $R$ is right perfect.

(2) Every flat right $R$-module is strongly Gorenstein flat.

In particular, if the class of strongly Gorenstein flat right $R$-modules is closed under direct limits, then $R$ is right perfect. 
ProOF. That (1) implies (2) is clear.

As to the converse, let $M$ be a flat right $R$-module. Then $M$ is projective by (2) and Corollary 2.5 , and so $R$ is right perfect.

If the class of strongly Gorenstein flat right $R$-modules is closed under direct limits, then any direct limit of projective right $R$-modules is both strongly Gorenstein flat and flat, and hence it is also projective by Corollary 2.5. Thus $R$ is right perfect by [4, Theorem P].

PROPOSITION 2.16. The following statements are equivalent for a ring $R$.

(1) $R$ is a $Q F$ ring (that is, $R$ is a 0 -Gorenstein ring).

(2) Every right $R$-module is strongly Gorenstein flat.

PROOF. This follows from the fact that $R$ is a $Q F$ ring if and only if injective right $R$-modules coincide with projective right $R$-modules.

PROPOSITION 2.17. The following statements are equivalent for a ring $R$.

(1) $R$ is an FC ring.

(2) Every (right and left) finitely presented R-module is strongly Gorenstein flat.

PROOF. To prove that (1) implies (2), let $M$ be a finitely presented right $R$-module and $F$ a flat right $R$-module. Then $F$ is $F P$-injective by [22, Lemma 4.1]. So $\operatorname{Ext}^{i}(M, F)=0$ for all $i \geq 1$. Thus $M$ is strongly Gorenstein flat by Theorem 2.6.

As to the converse, for any finitely presented right $R$-module $N, \operatorname{Ext}^{1}\left(N, R_{R}\right)=0$ since $N$ is strongly Gorenstein projective. So $R_{R}$ is $F P$-injective. Similarly, $R R$ is $F P$-injective. In addition, every finitely presented $R$-module has a $\mathcal{F} l a t$-pre-envelope. So $R$ is left and right coherent by [2, Proposition 2].

We conclude this section with some examples which show that strongly Gorenstein flat modules over coherent rings lie strictly between projective modules and Gorenstein flat modules.

EXAmple 2.18. Let $R=\mathbb{Z} / 4 \mathbb{Z}$, where $\mathbb{Z}$ is the ring of integers. Then $R$ is a commutative $Q F$ ring. It is clear that $2 R$ is a strongly Gorenstein flat $R$-module, but $2 R$ is not projective.

EXAMPLE 2.19. Let $R$ be a commutative coherent domain which is not a field, and let $Q$ denote the field of fractions of $R$. Then $Q$ is a (Gorenstein) flat $R$-module which is not contained in any free $R$-module. So $Q$ is not a strongly Gorenstein flat $R$-module by definition.

EXAMPLE 2.20. Let $R$ be a trivial extension of $\mathbb{Z}$ by $\mathbb{Q} / \mathbb{Z}$, that is,

$$
R=\left\{\left(\begin{array}{cc}
m & x \\
0 & m
\end{array}\right) \mid m \in \mathbb{Z}, x \in \mathbb{Q} / \mathbb{Z}\right\}
$$

with addition and multiplication as in ordinary matrices. Then $R$ is a commutative $F C$ ring (see $[9$, Example 1$])$. But $R / \operatorname{Rad}(R)=R /(0, \mathbb{Q} / \mathbb{Z}) \cong \mathbb{Z}$, where $\operatorname{Rad}(R)$ is the Jacobson radical of $R$. So $R$ is neither a von Neumann regular ring nor a perfect 
ring. Thus there exists a strongly Gorenstein flat $R$-module which is not projective (flat) by Proposition 2.14, and there exists a (Gorenstein) flat $R$-module which is not strongly Gorenstein flat by Proposition 2.15.

\section{Strongly Gorenstein flat dimension}

In this section, we define and investigate the strongly Gorenstein flat dimension for modules and rings.

Definition 3.1. For a right $R$-module $M$, let $S G f d(M)$ denote the infimum of the set of $n$ such that there exists an exact sequence $0 \rightarrow G_{n} \rightarrow \cdots \rightarrow G_{1} \rightarrow G_{0} \rightarrow M \rightarrow$ 0 of right $R$-modules, where each $G_{i}$ is strongly Gorenstein flat and call $\operatorname{SGfd}(M)$ the strongly Gorenstein flat dimension of $M$. If no such $n$ exists, set $S G f d(M)$ $=\infty$. Put $r S G F D(R)$ equal to the supremum of the set of $S G f d(M)$ such that $M$ is any right $R$-module and $r F I D(R)$ equal to the supremum of the set of $\operatorname{id}(M)$ such that $M$ is any flat right $R$-module.

PROPOSITION 3.2. Let $R$ be a ring. Then $r F I D(R) \leq r S G F D(R) \leq r D(R)$. The equalities hold if $w D(R)<\infty$.

PRoOF. It is clear that $r S G F D(R) \leq r D(R)$. Let $r S G F D(R)=n<\infty$ and $F$ be a flat right $R$-module. For any right $R$-module $N$, there is an exact sequence $0 \rightarrow P_{n} \rightarrow P_{n-1} \rightarrow \cdots \rightarrow P_{1} \rightarrow P_{0} \rightarrow N \rightarrow 0$ with each $P_{i}$ strongly Gorenstein flat by assumption. It follows that $\operatorname{Ext}^{n+i}(N, F) \cong \operatorname{Ext}^{i}\left(P_{n}, F\right)=0$ for all $i \geq 1$ by Lemma 2.4(1). Thus $\mathrm{id}(F) \leq n$, and so $r F I D(R) \leq r S G F D(R)$.

Let $w D(R)<\infty$. We only need to show that $r D(R) \leq r F I D(R)$. In fact, we may assume that $r F I D(R)=m<\infty$. For any right $R$-module $M$, there exist a nonnegative integer $k$ and an exact sequence $0 \rightarrow F_{k} \rightarrow F_{k-1} \rightarrow \cdots \rightarrow F_{1} \rightarrow F_{0} \rightarrow$ $M \rightarrow 0$ with each $F_{i}$ flat since $\operatorname{fd}(M)<\infty$. Note that $\operatorname{id}\left(F_{i}\right) \leq m$, and so $\operatorname{id}(M) \leq m$. Thus $r D(R) \leq r F I D(R)$, and hence the equalities follow.

REMARK 3.3. Clearly, $r S G F D(R)=0$ if and only if $R$ is a $Q F$ ring, which holds if and only if $r F I D(R)=0$. For example, $R=\mathbb{Z} / 4 \mathbb{Z}$ is a $Q F$ ring, and so $r F I D(R)$ $=r S G F D(R)=0$. But $r D(R)=w D(R)=\infty$.

LEMMA 3.4. Let $R$ be a left coherent ring and $n$ a fixed nonnegative integer. Consider the following conditions for a right $R$-module $M$.

(1) $S G f d(M) \leq n$.

(2) For any exact sequence $0 \rightarrow K_{n} \rightarrow P_{n-1} \rightarrow \cdots \rightarrow P_{1} \rightarrow P_{0} \rightarrow M \rightarrow 0$ with each $P_{i}$ projective, $K_{n}$ is strongly Gorenstein flat.

(3) $\operatorname{Ext}^{n+i}(M, F)=0$ for any flat right $R$-module $F$ and any $i \geq 1$.

Then (1) and (2) are equivalent and imply (3). Moreover, (3) implies (2) if F P. $\operatorname{id}\left({ }_{R} R\right)<\infty$.

Proof. By (1), there exists an exact sequence $0 \rightarrow G_{n} \rightarrow \cdots \rightarrow G_{1} \rightarrow G_{0} \rightarrow M \rightarrow$ 0 of right $R$-modules with each $G_{i}$ strongly Gorenstein flat. By Proposition 2.10 and [3, Lemma 3.12], $K_{n}$ is strongly Gorenstein flat, thus (1) implies (2).

That (2) implies (1) is clear by definition. 
For any flat right $R$-module $F$ and any $i \geq 1$, we have $\operatorname{Ext}^{n+i}(M, F)$ $\cong \operatorname{Ext}^{i}\left(K_{n}, F\right)=0$ by (2) and Lemma 2.4(1), thus (2) implies (3).

That (3) implies (2) holds by Theorem 2.6.

COROLLARY 3.5. The following statements are true for a ring $R$.

(1) If $R$ is a left coherent ring and $F P-\mathrm{id}\left({ }_{R} R\right)<\infty$, then $r S G F D(R)=r F I D(R)$.

(2) If $R$ is an $n$-Gorenstein ring, then $r S G F(R)=r F I D(R) \leq n$.

PROOF. (1) holds by Lemma 3.4; (2) comes from (1) and [13, Proposition 9.1.2].

We are now in a position to give the main result of this section.

THEOREM 3.6. The following statements are equivalent for a two-sided coherent ring $R$ and a fixed integer $n \geq 1$.

(1) $R$ is an $n-F C$ ring.

(2) $S G f d(M) \leq n$ for any (right and left) finitely presented $R$-module $M$.

(3) The nth $\mathcal{F l a t - c o s y z y g y ~ o f ~ e v e r y ~ ( r i g h t ~ a n d ~ l e f t ) ~ f i n i t e l y ~ p r e s e n t e d ~} R$-module is strongly Gorenstein flat.

Proof. To show that (1) implies (2), let $M$ be a finitely presented $R$-module and $F$ be a flat $R$-module. Then $F P-\operatorname{id}(F) \leq n$ by (1) and [13, Theorem 8.4.31], and so $\operatorname{Ext}^{n+i}(M, F)=0$ for all $i \geq 1$. Thus $\operatorname{SG} f d(M) \leq n$ by Lemma 3.4.

We now prove that (2) implies (1). For any finitely presented right $R$-module $M$, $\operatorname{Ext}^{n+1}\left(M, R_{R}\right)=0$ by (2) and Lemma 3.4. So $F P$-id $\left(R_{R}\right) \leq n$. Similarly, it follows that $F P-\operatorname{id}\left({ }_{R} R\right) \leq n$.

To show that (1) implies (3), let $M$ be a finitely presented $R$-module and $0 \rightarrow M \rightarrow P^{0} \rightarrow P^{1} \rightarrow \cdots$ be a right $\mathcal{F}$ lat-resolution of $M$ with each $P_{i}$ finitely generated projective and $L^{n}$ the $n$th $\mathcal{F}$ lat-cosyzygy. Then the sequence is exact at $P^{k}$ for $k \geq n-1$ by [13, Theorem 8.4.31]. So we get an exact sequence

$$
0 \rightarrow L^{n} \rightarrow P^{n} \rightarrow \cdots \rightarrow P^{2 n-1} \rightarrow L^{2 n} \rightarrow 0 .
$$

Note that $L^{n}$ and $L^{2 n}$ are finitely presented. Let $F$ be a flat $R$-module. Evidently $F P-\operatorname{id}(F) \leq n$ by [13, Theorem 8.4.31]. So $\operatorname{Ext}^{i}\left(L^{n}, F\right) \cong \operatorname{Ext}^{n+i}\left(L^{2 n}, F\right)=0$ for all $i \geq 1$. Thus $L^{n}$ is strongly Gorenstein flat by Theorem 2.6.

Finally, to show that (3) implies (1), let $N$ be a finitely presented right $R$-module. Then $N$ admits a right $\mathcal{F l a t}$-resolution

$$
\mathcal{E}: 0 \rightarrow N \rightarrow P^{0} \rightarrow P^{1} \rightarrow \cdots
$$

Note that the $n$th $\mathcal{F l a t - c o s y z y g y} L^{n}$ is strongly Gorenstein flat by (3). So there is a $\operatorname{Hom}(-, \mathcal{F}$ lat $)$ exact exact sequence $0 \rightarrow L^{n} \rightarrow F^{1} \rightarrow F^{2} \rightarrow \cdots$ with each $F^{i}$ projective. Thus $\mathcal{E}$ is exact at $P^{k}$ for $k \geq n-1$. Hence $F P-\operatorname{id}\left({ }_{R} R\right) \leq n$ by [13, Theorem 8.4.31]. Similarly, $F P-\operatorname{id}\left(R_{R}\right) \leq n$. 


\section{Precovers and pre-envelopes by strongly Gorenstein flat modules}

In this section, we study the existence of strongly Gorenstein flat precovers and preenvelopes. Let $\mathcal{S G \mathcal { F }}$ be the class of all strongly Gorenstein flat right $R$-modules.

THEOREM 4.1. Let $R$ be a left coherent ring and $n$ be a fixed nonnegative integer with

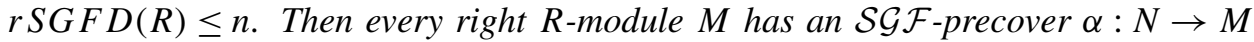
such that if $0 \rightarrow K \rightarrow N \stackrel{\alpha}{\rightarrow} M \rightarrow 0$ is exact, then $\operatorname{pd}(K) \leq n-1$ whenever $n \geq 1$. Moreover, if $\operatorname{pd}(M)<\infty$, then $N$ is projective.

PRoOF. By hypothesis, there is an exact sequence $0 \rightarrow K_{n} \rightarrow P_{n-1} \rightarrow \cdots \rightarrow P_{1} \rightarrow$ $P_{0} \rightarrow M \rightarrow 0$ such that each $P_{i}$ is projective and $K_{n}$ is strongly Gorenstein flat by Lemma 3.4 since $S G f d(M) \leq n$. Then there exists a $\operatorname{Hom}(-, \mathcal{F l a t})$ exact exact sequence $0 \rightarrow K_{n} \rightarrow P^{0} \rightarrow \cdots \rightarrow P^{n-2} \rightarrow P^{n-1} \rightarrow L^{n} \rightarrow 0$ with each $P^{i}$ projective. Note that $L^{n}$ is strongly Gorenstein flat. So the following diagram commutes:

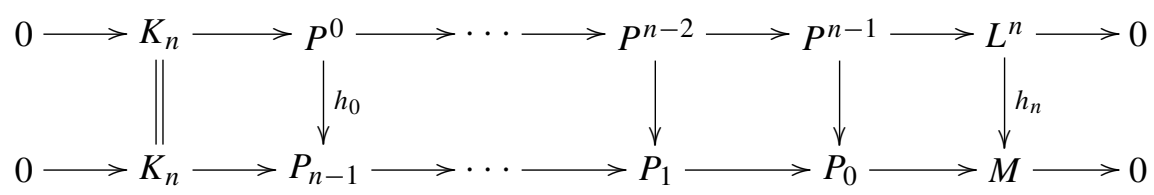

By [13, Proposition 1.4.14], we get an exact sequence

$$
0 \rightarrow K_{n} \rightarrow P^{0} \oplus K_{n} \rightarrow P^{1} \oplus P_{n-1} \rightarrow \cdots \rightarrow P^{n-1} \oplus P_{1} \rightarrow L^{n} \oplus P_{0} \rightarrow M \rightarrow 0
$$

which gives the exactness of the sequence

$$
0 \rightarrow P^{0} \rightarrow P^{1} \oplus P_{n-1} \rightarrow \cdots \rightarrow P^{n-1} \oplus P_{1} \rightarrow L^{n} \oplus P_{0} \rightarrow M \rightarrow 0 .
$$

Let $N=L^{n} \oplus P_{0}$. Consider the short exact sequence $0 \rightarrow K \rightarrow N \rightarrow M \rightarrow 0$. It is clear that $\operatorname{pd}(K) \leq n-1$ and $N$ is strongly Gorenstein flat. So $\operatorname{Ext}^{1}(H, K)=0$ for any strongly Gorenstein flat right $R$-module $H$ by Lemma 2.4(1). Thus $N \rightarrow M$ is an $\mathcal{S G} \mathcal{F}$-precover.

If $\operatorname{pd}(M)<\infty$, then $\operatorname{pd}(N)<\infty$ since $\operatorname{pd}(K)<\infty$. It follows that $N$ is projective by Lemma 2.4(2).

Let $\mathcal{G P}$ be the class of all Gorenstein projective right $R$-modules and $\mathcal{I}_{n}$ the class of all right $R$-modules of injective dimension at most $n$, where $n$ is a fixed nonnegative integer. Enochs and Jenda proved that $\left(\mathcal{G P}, \mathcal{I}_{n}\right)$ is a cotorsion theory if $R$ is an $n$-Gorenstein ring (see [13, Remark 11.5.10]). This result is a particular case of the following theorem by Corollaries 2.8 and 3.5(2).

THEOREM 4.2. Let $R$ be a left coherent ring, $n$ be a fixed nonnegative integer and $F P-\operatorname{id}\left({ }_{R} R\right) \leq n$. Then the following statements are equivalent.

(1) $\quad r S G F D(R) \leq n$.

(2) $\quad r F I D(R) \leq n$.

(3) $\left(\mathcal{S G F}, \mathcal{I}_{n}\right)$ is a cotorsion theory. 
ProOf. The case $n=0$ is clear. Next we assume that $n \geq 1$.

That (1) holds if and only if (2) holds follows from Corollary 3.5(1).

To show that (2) implies (3), let $E$ be an injective right $R$-module. Then there is a split exact sequence $0 \rightarrow E \rightarrow \Pi\left({ }_{R} R\right)^{+}$since $\left({ }_{R} R\right)^{+}$is a cogenerator. Since $R$ is left coherent, $\operatorname{fd}\left(\left({ }_{R} R\right)^{+}\right)=F P-\mathrm{id}\left({ }_{R} R\right) \leq n$, and so $\mathrm{fd}\left(\Pi\left({ }_{R} R\right)^{+}\right) \leq n$. Hence $\operatorname{fd}(E) \leq n$. Thus $\operatorname{fd}(Q)<\infty$ for any $Q \in \mathcal{I}_{n}$. So $\mathcal{I}_{n} \subseteq \mathcal{S G \mathcal { F }}{ }^{\perp}$ and $\mathcal{S G \mathcal { F }} \subseteq{ }^{\perp} \mathcal{I}_{n}$ by Lemma 2.4(1).

Let $M$ be a right $R$-module such that $M \in \mathcal{S G \mathcal { F }}{ }^{\perp}$, that is, $\operatorname{Ext}^{1}(N, M)=0$ for all strongly Gorenstein flat right $R$-modules $N$. Then there is an exact sequence $0 \rightarrow M \rightarrow E \rightarrow L \rightarrow 0$ with $E$ injective. Note that (2) is equivalent to (1), so there exists an exact sequence $0 \rightarrow K \rightarrow G \rightarrow L \rightarrow 0$ with $G$ strongly Gorenstein flat and $\operatorname{pd}(K) \leq n-1$ by Theorem 4.1. Then we obtain the following pullback diagram:

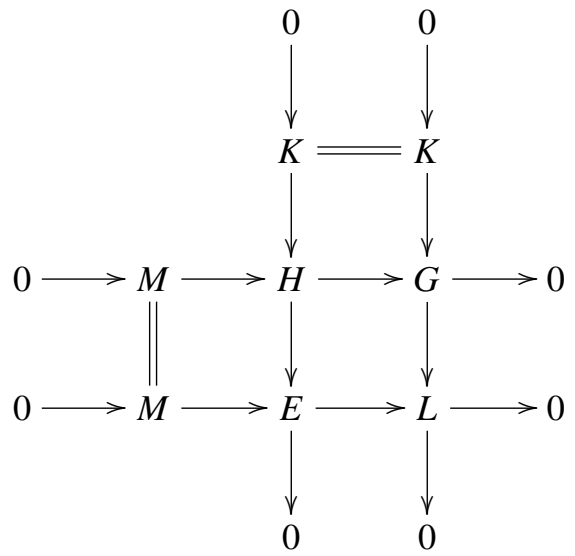

Since $\operatorname{Ext}^{1}(G, M)=0$, the sequence $0 \rightarrow M \rightarrow H \rightarrow G \rightarrow 0$ is split. Note that $\operatorname{id}(H) \leq n$ since $\operatorname{id}(K) \leq n$ by (2). Thus $\operatorname{id}(M) \leq n$, and so $\mathcal{S G \mathcal { F }} \mathcal{F}^{\perp} \subseteq \mathcal{I}_{n}$. Hence $\mathcal{S G} \mathcal{F}^{\perp}=\mathcal{I}_{n}$.

Now let $N \in{ }^{\perp} \mathcal{I}_{n}$, that is, $\operatorname{Ext}^{1}(N, F)=0$ for all right $R$-modules $F$ such that $\operatorname{id}(F) \leq n$. By Theorem 4.1, we get an exact sequence $0 \rightarrow A \rightarrow B \rightarrow N \rightarrow 0$ with $B$ strongly Gorenstein projective and $\operatorname{pd}(A) \leq n-1$. So the sequence is split since $\operatorname{id}(A) \leq n$ by (2), and hence $N$ is strongly Gorenstein flat. Thus ${ }^{\perp} \mathcal{I}_{n} \subseteq \mathcal{S G \mathcal { F }}$, and hence ${ }^{\perp} \mathcal{I}_{n}=\mathcal{S} \mathcal{G} \mathcal{F}$. Therefore, $\left(\mathcal{S G \mathcal { F }}, \mathcal{I}_{n}\right)$ is a cotorsion theory.

Finally, to show that (3) implies (1), let $M$ be any right $R$-module. There is an exact sequence $0 \rightarrow K_{n} \rightarrow P_{n-1} \rightarrow \cdots \rightarrow P_{1} \rightarrow P_{0} \rightarrow M \rightarrow 0$ with each $P_{i}$ projective. Since $\operatorname{Ext}^{1}\left(K_{n}, H\right) \cong \operatorname{Ext}^{n+1}(M, H)=0$ for any $R$-module $H$ with $\operatorname{id}(H) \leq n$, $K_{n}$ is strongly Gorenstein flat by (3). Thus $\operatorname{SGfd}(M) \leq n$ by Lemma 3.4, and so $r S G F D(R) \leq n$.

THEOREM 4.3. Let $R$ be a left coherent ring and $n$ a fixed nonnegative integer such that $S G F d(N) \leq n$ for any finitely presented right $R$-module $N$. Then every finitely

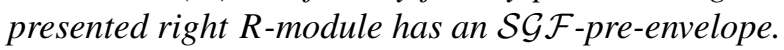


Proof. Let $M$ be a finitely presented right $R$-module. Then there is a $\operatorname{Hom}(-, \mathcal{F}$ lat $)$ exact complex $0 \rightarrow M \rightarrow F^{0} \rightarrow F^{1} \rightarrow \cdots \rightarrow F^{n-1} \rightarrow L^{n} \rightarrow 0$ with each $F_{i}$ finitely generated projective since $R$ is left coherent. Note that there exists an exact sequence $0 \rightarrow K_{n} \rightarrow P_{n-1} \rightarrow \cdots \rightarrow P_{1} \rightarrow P_{0} \rightarrow L^{n} \rightarrow 0$ with each $P_{i}$ projective. Thus $K_{n}$ is strongly Gorenstein flat by Lemma 3.4 since $S G F d\left(L^{n}\right) \leq n$. For any strongly Gorenstein flat right $R$-module $G$ and any homomorphism $f: M \rightarrow G$, there is a $\operatorname{Hom}(-, \mathcal{F}$ lat $)$ exact exact sequence $0 \rightarrow G \rightarrow H^{0} \rightarrow H^{1} \rightarrow \cdots \rightarrow H^{n-1} \rightarrow Q^{n} \rightarrow$ 0 with each $H^{i}$ projective, and so we obtain the following commutative diagram:

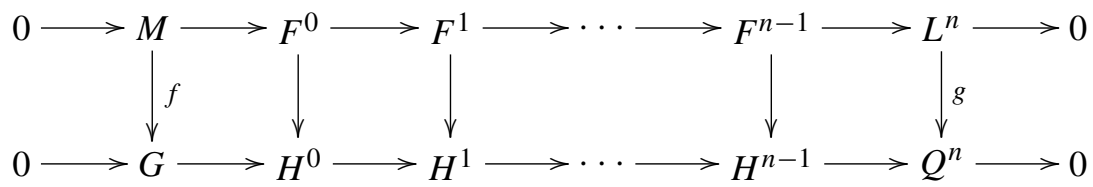

But we also have the following commutative diagram:

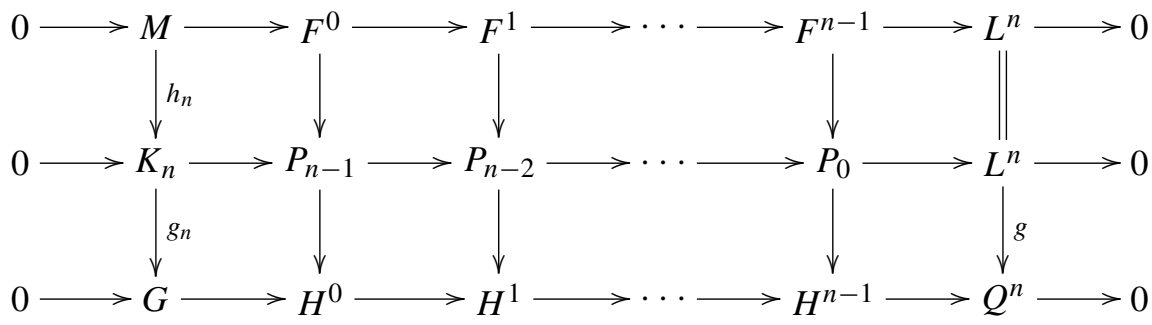

Then the usual homotopy argument shows that $f-g_{n} h_{n}$ factors through $F^{0}$. So $f: M \rightarrow G$ factors through the strongly Gorenstein flat right $R$-module $K_{n} \oplus F^{0}$.

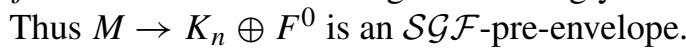

Recall that a left $R$-module $M$ is called Gorenstein injective [12] if there is an exact sequence $\cdots \rightarrow E_{1} \rightarrow E_{0} \rightarrow E^{0} \rightarrow E^{1} \rightarrow \cdots$ of injective left $R$-modules with $M=\operatorname{ker}\left(E^{0} \rightarrow E^{1}\right)$ such that $\operatorname{Hom}(E,-)$ leaves the sequence exact whenever $E$ is an injective left $R$-module.

The following theorem is a generalization of [13, Corollary 11.8.3].

THEOREM 4.4. Let $R$ be an $n-F C$ ring. Then every finitely presented right $R$-module

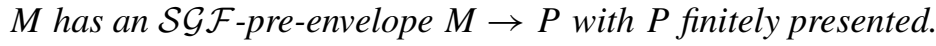

PROOF. The result can be obtained as a corollary of Theorems 3.6 and 4.3. Here we give another proof which may be of independent interest.

Let $M$ be a finitely presented right $R$-module. By [13, Lemma 5.3.12], there is a cardinal number $\aleph_{\alpha}$ such that for any homomorphism $f: M \rightarrow L$ with $L$ Gorenstein flat, there is a pure submodule $Q$ of $L$ such that $\operatorname{Card}(Q) \leq \aleph_{\alpha}$ and $f(M) \subseteq Q$. We claim that $Q$ is Gorenstein flat. In fact, the pure exact sequence $0 \rightarrow Q \rightarrow L \rightarrow$ $L / Q \rightarrow 0$ induces a split exact sequence $0 \rightarrow(L / Q)^{+} \rightarrow L^{+} \rightarrow Q^{+} \rightarrow 0$. Since $L^{+}$is Gorenstein injective by [20, Theorem 3.6], $Q^{+}$is Gorenstein injective by 
[20, Theorem 2.6]. Thus $Q$ is Gorenstein flat by [20, Theorem 3.6] again. It follows that $f$ has a factorization $M \rightarrow Q \rightarrow L$ with $\operatorname{Card}(Q) \leq \aleph_{\alpha}$ and $Q$ Gorenstein flat. Now let $\left(\varphi_{i}\right)_{i \in I}$ give all such homomorphisms $\varphi_{i}: M \rightarrow Q_{i}$. So any homomorphism $M \rightarrow H$ with $H$ Gorenstein flat has a factorization $M \rightarrow Q_{j} \rightarrow H$ for some $j \in I$. Thus the homomorphism $\varphi: M \rightarrow \prod_{i \in I} Q_{i}$ induced by all $\varphi_{i}$ is a Gorenstein Flatpre-envelope since $\prod_{i \in I} Q_{i}$ is Gorenstein flat by [10, Corollary 8]. But $M$ is finitely presented, so $\varphi$ factors through a finitely presented strongly Gorenstein flat right $R$ module $P$ by Lemma 2.13 and [10, Theorem 5], that is, there exist homomorphisms $g: M \rightarrow P$ and $h: P \rightarrow \prod_{i \in I} Q_{i}$ such that $\varphi=h g$. It is easy to verify that $g$ is an $\mathcal{S G} \mathcal{F}$-pre-envelope of $M$.

We round off the paper with the following remark which may be viewed as an illustration of the usefulness of strongly Gorenstein flat modules.

REMARK 4.5. (1) If $R$ is a left coherent ring, then every projective module is strongly Gorenstein flat, and every strongly Gorenstein flat module is Gorenstein flat, but no two of these concepts are equivalent by Proposition 2.3 and Examples 2.18, 2.19 and 2.20 .

(2) If $R$ is an $n$-Gorenstein ring, then strongly Gorenstein flat modules are precisely Gorenstein projective modules by Corollary 2.8 .

(3) By definition, every strongly Gorenstein flat module is Gorenstein projective, but we have not been able to find examples of Gorenstein projective modules which are not strongly Gorenstein flat. If one can find a Gorenstein projective module $M$ over a coherent ring which is not Gorenstein flat, then $M$ is not strongly Gorenstein flat by Proposition 2.3.

(4) Holm [19] asked whether every Gorenstein projective module is Gorenstein flat. This question remains open, but it is now known that if $R$ is a left coherent ring with finite right finitistic projective dimension (or $R$ is a commutative Noetherian ring with finite Krull dimension), then every Gorenstein projective right $R$-module is Gorenstein flat (see [19, Proposition 5.5] and [20, Proposition 3.4]).

(5) If the class of strongly Gorenstein flat modules happens to be the class of Gorenstein projective modules, then Proposition 2.3 gives an affirmative answer to Holm's question for any left coherent ring.

\section{Acknowledgement}

Part of this work was done while the first author was visiting Brock University at St. Catharines, Canada. He would like to thank the Department of Mathematics of Brock University for its kind hospitality.

\section{References}

[1] F. W. Anderson and K. R. Fuller, Rings and Categories of Modules (Springer, Berlin, 1974).

[2] J. Asensio Mayor and J. Martinez Hernandez, 'Flat envelopes in commutative rings', Israel J. Math. 62 (1988), 123-128. 
[3] M. Auslander and M. Bridge, Stable Module Theory, Memoirs of the American Mathematical Society, 94 (American Mathematical Society, Providence, RI, 1969).

[4] H. Bass, 'Finitistic Dimension and a homological generalization of semi-primary rings', Trans. Amer. Math. Soc. 95 (1960), 466-488.

[5] D. Bennis and N. Mahdou, 'Strongly Gorenstein projective, injective, and flat modules', J. Pure Appl. Algebra 210 (2007), 437-445.

[6] L. Bican, R. El Bashir and E. E. Enochs, 'All modules have flat covers', Bull. London Math. Soc. 33 (2001), 385-390.

[7] L. W. Christensen, Gorenstein Dimensions, Lecture Notes in Mathematics, 1747 (Springer, Berlin, 2000).

[8] L. W. Christensen, A. Frankild and H. Holm, 'On Gorenstein projective, injective and flat dimension - a functorial description with applications', J. Algebra 302 (2006), 231-279.

[9] R. R. Colby, 'Rings which have flat injective modules', J. Algebra 35 (1975), 239-252.

[10] N. Q. Ding and J. L. Chen, 'Coherent rings with finite self-F P-injective dimension', Comm. Algebra 24 (1996), 2963-2980.

[11] E. E. Enochs, 'Injective and flat covers, envelopes and resolvents', Israel J. Math. 39 (1981), 189-209.

[12] E. E. Enochs and O. M. G. Jenda, 'Gorenstein injective and Gorenstein projective modules', Math. Z. 220 (1995), 611-633.

[13] Relative Homological Algebra (Walter de Gruyter, Berlin, 2000).

[14] E. E. Enochs, O. M. G. Jenda and J. A. López-Ramos, 'The existence of Gorenstein flat covers', Math. Scand. 94 (2004), 46-62.

[15] E. E. Enochs, O. M. G. Jenda and B. Torrecillas, 'Gorenstein flat modules', Nanjing Daxue Xuebao Shuxue Bannian Kan 10 (1993), 1-9.

[16] E. E. Enochs and J. A. López-Ramos, Gorenstein Flat Modules (Nova Science Publishers, New York, 2001).

[17] D. J. Fieldhouse, 'Character modules, dimension and purity', Glasgow Math. J. 13 (1972), 144-146.

[18] S. Glaz, Commutative Coherent Rings, Lecture Notes in Mathematics, 1371 (Springer, Berlin, 1989).

[19] H. Holm, 'Gorenstein projective, injective and flat modules', MSc Thesis, Institute for Mathematical Sciences, University of Copenhagen, 2000.

[20] - 'Gorenstein homological dimensions', J. Pure Appl. Algebra 189 (2004), 167-193.

[21] J. J. Rotman, An Introduction to Homological Algebra (Academic Press, New York, 1979).

[22] B. Stenström, 'Coherent rings and FP-injective modules', J. London Math. Soc. 2 (1970), 323-329.

[23] J. Xu, Flat Covers of Modules, Lecture Notes in Mathematics, 1634 (Springer, Berlin, 1996).

NANQING DING, Department of Mathematics, Nanjing University, Nanjing 210093, PR China

e-mail: nqding@nju.edu.cn

YUANLIN LI, Department of Mathematics, Brock University, St. Catharines, Ontario, Canada L2S 3A1

e-mail: yli@brocku.ca

LIXIN MAO, Institute of Mathematics, Nanjing Institute of Technology, Nanjing 211167, PR China e-mail: maolx2@hotmail.com 